\title{
Studies on photoreactive and biodegradable copolymers composed of poly( $\varepsilon$-caprolactone) and 4-hydroxycinnamic acid
}

\author{
Jihang Li, Huaqing Xu, Na Hu, Dongjian Shi, Weifu Dong, Chao Wu and Mingqing Chen
}

A novel group of photoreactive and biodegradable copolymers known as poly( $\varepsilon$-caprolactone)-co-poly(4-hydroxycinnamic acid) (PCL-co-P4HCA) was obtained by the thermal melt polycondensation of poly( $\varepsilon$-caprolactone) (PCL) with 4-hydroxycinnamic acid. The chemical structures of the PCL-co-P4HCA copolymers were analyzed by Fourier transform infrared spectroscopy and nuclear magnetic resonance. The melting points of the PCL-co-P4HCA copolymers were dependent on the PCL and 4HCA compositions, as observed by differential scanning calorimetry measurements. Wide-angle X-ray diffraction (WXRD) analysis showed highly crystalline structures of the P4HCA, PCL and PCL-co-P4HCA polymers. The PCL-co-P4HCA copolymers were fluorescent and had good photoreactivities. Crosslinking of the cinnamoyl groups in the copolymers via ultraviolet (UV) irradiation significantly affected the fluorescence, thermal stability, hydrophobicity and water absorption of the resultant copolymers. The PCL-co-P4HCA copolymers underwent degradation when placed in buffer solutions at $\mathrm{pH} 7.4$ and $\mathrm{pH} 13.3$, and UV irradiation was able to regulate the degradation process. The photoreactive and biodegradable copolymers with high thermal stability may be potentially applied in both the biomedical and environmental fields.

Polymer Journal (2012) 44, 1123-1130; doi:10.1038/pj.2012.85; published online 23 May 2012

Keywords: biodegradability; crosslinking; 4-hydroxycinnamic acid; photoreactivity; poly(e-caprolactone)

\section{INTRODUCTION}

The development of biodegradable polymers derived from nature monomers is considered to be an effective method of solving environmental and resource problems. ${ }^{1,2}$ Polylactide, $\operatorname{poly}(\varepsilon-$ caprolactone) (PCL), poly(hydroxyalkanoate) and poly(butylenes succinate) are widely known to be environmentally degradable polymers. PCL is a type of aliphatic polyester typically prepared by the ring-opening polymerization of $\varepsilon$-caprolactone $(\varepsilon-\mathrm{CL})$ using the primary hydroxyl groups of an alcohol $(\mathrm{ROH})$ or $\mathrm{H}_{2} \mathrm{O}$ as initiators. PCL is widely utilized in numerous biomedical applications such as sustained-release drug-delivery systems and tissue-engineering scaffolds because of its excellent biocompatibility and biodegradability. $^{3-6}$ Nevertheless, the applications of PCL for biomedical purposes are limited by its poor hydrophilicity, low melting temperature $\left(T_{\mathrm{m}}\right)$, low mechanical properties and slow degradation rate. Therefore, it is necessary to improve its properties for increasing the scope of its applicability. One of the most effective methods for improving the properties of PCL is to copolymerize with other monomers or polymers. There have been recent reports documenting the copolymerization of $\varepsilon$-CL or PCL with other materials such as lactide, acrylic acid, $N$-isopropylacrylamide (NIPAAm) and poly(ethylene glycol), ${ }^{4,71}$ Although the solubility and degradation rate of the PCL copolymers were enhanced, the mechanical and thermal properties still require further improvements. Generally, the introduction of aromatic polymers with rigid benzene ring components into aliphatic polymers such as PCL is an efficient method to significantly improve the mechanical and thermal properties of these aliphatic polymers. Moreover, functional materials that respond to environmental stimuli, such as photoresponsive materials, can be introduced into PCL to widen its applicability in tissue engineering, controlled drug-delivery systems and other fields.

4-hydroxycinnamic acid (4HCA) is a derivative of coumaric acid that exists in plant cell walls as an intermediate metabolite of lignin and other biological materials. It is a typical biomonomer, and its photoreactivities involving an addition reaction with $[2+2]$ cyclobutane and other trans-cis transformations are notable. ${ }^{12}$ The homopolymer of 4HCA, P4HCA, has good photoreactivity, cell compatibility, degradability, liquid crystallinity and thermal properties, but it also exhibits poor solubility and is a brittle material. Akashi and coworkers selected 3,4-dihydroxycinnamic acid, lactic acid, cholic acid and lithocholic acid for copolymerization tests with 4HCA, and the resultant copolymers showed high solubilities and thermal and mechanical properties in addition to excellent photoreactivities, biocompatibilities and biodegradabilities. ${ }^{13-16}$ However, the influences of UV irradiation 
on the thermal and fluorescence properties of these polymers have not yet been investigated.

To increase the thermal and stimuli response properties of the PCL polymer, a novel photoreactive and biodegradable copolymer known as poly( $\varepsilon$-caprolactone)-co-poly(4-hydroxycinnamic acid) (PCL-coP4HCA) was prepared in this paper. The copolymers are expected to possess both the characteristics of PCL and P4HCA, such as thermal stability, photoreactivity, fluorescence and biodegradability. The effects of the monomer composition and UV irradiation on the degradable, photoreactive, thermal and fluorescence properties of the copolymers were investigated in detail. Moreover, the thermal properties before and after UV irradiation were analyzed and are specifically discussed in this paper. The PCL-co-P4HCA copolymers have good biocompatibility and low toxicity and can therefore be used in biomedical, drug-controlled release and fluorescent probe fields.

\section{EXPERIMENTAL PROCEDURE}

\section{Materials}

4HCA monomer was purchased from Wuhan Yuancheng Technology Development Co. Ltd., Wuhan, Hubei, China, and purified in $\mathrm{DMF} / \mathrm{H}_{2} \mathrm{O}$ $(\mathrm{v} / \mathrm{v}=1: 50)$ before use. $\varepsilon$-CL was purchased from Acros Organics (Geel, Belgium) and was used without further purification. Anhydride acetic acid $\left(\mathrm{Ac}_{2} \mathrm{O}\right)$, sodium acetate $(\mathrm{NaOAc})$, tetrahydrofuran (THF), N,N-dimethylformamide (DMF), dichloromethane (DCM) and methanol were purchased from a Shanghai Chemical Reagent Stationsed (Shanghai, China) directly.

\section{Synthesis of PCL}

PCL was synthesized by a ring-opening polymerization method shown in Scheme 1. First, a dry round-bottom flask was filled with $\varepsilon$-CL under a nitrogenous atmosphere. A solution of $\mathrm{Sn}(\mathrm{oct})_{2}$ (containing $0.3 \mathrm{~mol} \%$ of $\varepsilon$-CL) in DCM was then added into the flask as a catalyst. The reaction mixture was heated at $110^{\circ} \mathrm{C}$ in vacuum for $8 \mathrm{~h}$. Later, the reaction mixture was cooled to room temperature, dissolved in a minimal amount of THF and precipitated by dropwise addition into methanol with continuous stirring. The resultant precipitate was redissolved in THF and reprecipitated into cold water. The final product was freeze-dried for $24 \mathrm{~h}$.

\section{Synthesis of PCL-co-P4HCA}

PCL-co-P4HCA copolymers were synthesized via a thermal polycondensation method shown in Scheme 2. 4HCA was mixed with a certain amount of PCL in a round-bottom flask containing $\mathrm{Ac}_{2} \mathrm{O}(30 \mathrm{ml})$ with $\mathrm{NaOAc}(1 \mathrm{~mol} \%$ of the monomers). After purging with dry nitrogen to remove moisture and oxygen in the flask, the mixture was placed in a silicon oil bath at $150{ }^{\circ} \mathrm{C}$ and stirred for $1 \mathrm{~h}$ until the solution became homogeneous. The reaction system was further heated to $200^{\circ} \mathrm{C}$ for an additional $6 \mathrm{~h}$. The viscosity of the solution increased gradually along with reaction time. The product was then cooled to room temperature and washed with ethanol twice. All the polycondensation reactions were carried out under shading and under a nitrogen atmosphere.

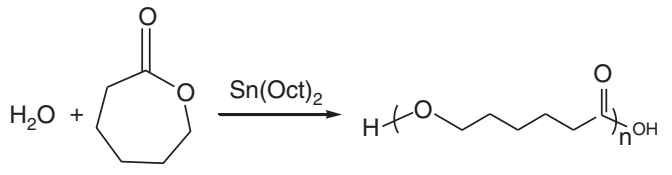

Scheme 1 Synthesis of PCL homopolymer.

\section{Preparation of the polymer sheet}

The polymer sheets $(30 \mathrm{~mm} \times 10 \mathrm{~mm}, 0.2 \mathrm{~mm}$ in thickness) were prepared by the melt-pressing method at $180^{\circ} \mathrm{C}$ at a pressure of $15 \mathrm{MPa}$ for $10 \mathrm{~min}$ and then cooled to room temperature.

\section{Characterization}

Fourier transform infrared spectroscopy (FTIR, FTLA2000, ABB Bomen, Quebec QC, Canada) and nuclear magnetic resonance $\left({ }^{1} \mathrm{H}\right.$ NMR, Bruker AVANCEIII, $400 \mathrm{MHz}$, Bruker Co., Fällanden, Switzerland) were used to identify the polymer structures and determine the monomeric compositions of the PCLco-P4HCA copolymers. A UV-visible spectrum (UV-1100, Beijing Rayleigh Analytical Instruments Co., Beijing, China) was used to determine the absorption of the PCL-co-P4HCA copolymers dissolved in THF. The solutions were irradiated by UV light $\left(\lambda=302 \mathrm{~nm}, 1 \mathrm{~mW} / \mathrm{cm}^{2}, \mathrm{UV}-100\right.$, Tanon Science And Technology Co. Ltd., Shanghai, China) for various times. The average molecular weight of the copolymers was determined by gel permeation chromatography (Aligent 1100, Santa Clara, CA, USA) using N,N-Dimethylformamide (DMF) as the eluent and polystyrene as the standard with a flow rate of $1 \mathrm{ml} \mathrm{min}{ }^{-1}$ at $37^{\circ} \mathrm{C}$. The thermal properties were analyzed by differential scanning calorimetry (DSC 822e, Mettler Toledo, Zörich, Switzerland) and thermogravimetric analysis (TGA/DSC1/1100SF, Mettler Toledo). DSC analysis was carried out by scanning the samples at a heating rate of $10{ }^{\circ} \mathrm{Cmin}^{-1}$ from 20 to $240{ }^{\circ} \mathrm{C}$. The melting temperatures $\left(T_{\mathrm{m}}\right)$ of the samples were obtained from the DSC curves of the second heating. The thermal degradation behavior of the samples was observed from the TGA curves by heating from 50 to $600{ }^{\circ} \mathrm{C}$ at a rate of $10^{\circ} \mathrm{Cmin}^{-1}$ under a nitrogen atmosphere with a flow rate of $\sim 50 \mathrm{ml} \mathrm{min}^{-1}$. X-ray diffraction (Bruker D8 Advance, Rheinstetten, Germany) was used to investigate the crystallinity of the polymers. All the samples were cooled from the molten state. The fluorescence spectra (Shimadzu RF-5301PC Kyoto, Japan) of the samples were measured at room temperature. The samples were dissolved in THF $\left(0.5 \mathrm{mg} \mathrm{ml}^{-1}\right)$ and the excitation and emission slit width were both set at $3 \mathrm{~nm}$. The contact angle of the sheets was measured using an OCA 40 instrument (Dataphysics Co., Ltd., Stuttgart, Germany) with ultrapure water droplets. Several droplets of water were placed on the sheet surface to determine the average value of the contact angle. Each sheet, regardless of UV irradiation, was then placed in a test tube (with a stopper) containing potassium chloride-sodium hydroxide buffered solution $(\mathrm{pH}=13.3)$ or phosphate buffered saline $(\mathrm{pH}=7.4)$ at $37^{\circ} \mathrm{C}$ for predetermined periods of time. The hydrolyzed sheets were washed thoroughly with distilled water at room temperature and dried at $40{ }^{\circ} \mathrm{C}$ for $24 \mathrm{~h}$. The degree of degradation was estimated from the weight percentage $\left(W_{\mathrm{r}}\right)$ of the remaining products, which was calculated using the following equation: $W_{\mathrm{r}}(\mathrm{wt} \%)=100 \times W_{\mathrm{t}} / W_{0}$, where $W_{0}$ and $W_{\mathrm{t}}$ are the weights of the dry sample before and after the degradation. The calculated weight percentage was the average of three experimental values

\section{RESULTS AND DISCUSSION}

\section{Synthesis of PCL and PCL-co-P4HCA polymers}

The PCL polymer was obtained by ring-opening method as shown in Scheme 1. The $M_{\mathrm{n}}$ and $M_{\mathrm{w}} / M_{\mathrm{n}}$ of PCL were $9.6 \times 10^{4}$ and 1.21 , respectively. PCL-co-P4HCA copolymers of varying compositions were synthesized from PCL and 4HCA (showed in Table 1), which introduced photoreactive groups into the main chains by the thermal polycondensation method, shown in Scheme 2. The average molecular weight of the PCL-co-P4HCA copolymers was also listed in Table 1.

\section{Chemical structures of the PCL-co-P4HCA copolymers}

The chemical structures of the PCL-co-P4HCA copolymers were analyzed by FTIR and ${ }^{1} \mathrm{H}$ NMR spectroscopy. Figure 1 shows the<smiles>CC(=O)Oc1ccc(/C=C/C(=O)COCCCCCC(=O)O)cc1</smiles>

Scheme 2 Synthesis of PCL-co-P4HCA copolymers. 
Table 1 Synthesis conditions of PCL-co-P4HCA polymers

\begin{tabular}{|c|c|c|c|c|c|c|c|}
\hline Sample & $4 H C A(m m o l)$ & $C L(m m o l)$ & 4HCA:CL $(m o l \%)$ & $4 H C A: C L^{\mathrm{b}}$ (mol\%) & $M_{n} \times 10^{-4}\left(\mathrm{~g} \mathrm{~mol}^{-1}\right)^{c}$ & $\mathrm{M}_{w} \mathrm{M}_{n}$ & Yield (\%) \\
\hline PCL-co-P4HCA100 & 40 & 0 & 100.0 & 100.0 & 0.7 & 1.06 & 87.4 \\
\hline PCL-co-P4HCA75 & 30 & 7.5 & $80: 20$ & $75: 25$ & 8.7 & 1.10 & 86.3 \\
\hline PCL-CO-P4HCA33 & 20 & 30 & $40: 60$ & $33: 67$ & 10.1 & 1.22 & 85.9 \\
\hline PCL-co-P4HCA12 & 7.5 & 30 & $20: 80$ & $12: 88$ & 9.5 & 1.20 & 82.8 \\
\hline
\end{tabular}

Abbreviation: $\mathrm{CL}$, caprolactone.

a Molar ratios were calculated by amounts of the feed monomers.

${ }^{\mathrm{b}}$ Molar ratios were estimated by ${ }^{1} \mathrm{H}$ NMR spectra.

${ }^{\mathrm{C}}$ As measured by GPC with DMF as an eluent.

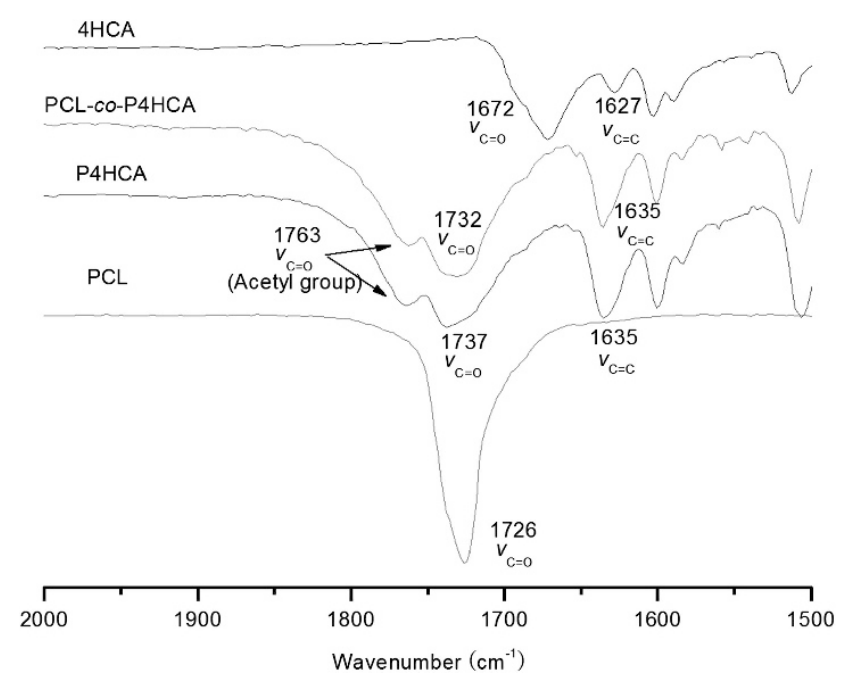

Figure 1 FTIR spectra of 4HCA, PCL-Co-P4HCA, P4HCA and PCL polymers. A full color version of this figure is available at Polymer Journal online.

FTIR spectra of the 4HCA, the PCL-co-P4HCA copolymer, the P4HCA and the PCL homopolymers. It can be observed that the $\mathrm{C}=\mathrm{O}$ stretching peak corresponding to carboxylic acid groups at $1672 \mathrm{~cm}^{-1}$ in the spectrum of the 4HCA monomer was absent from the spectra of the PCL-co-P4HCA and P4HCA polymers, while carbonyl $(\mathrm{C}=\mathrm{O})$ stretching bands of the ester group at $1732-1763 \mathrm{~cm}^{-1}$ appeared in the spectra of the PCL-co-P4HCA and P4HCA polymers, suggesting that carboxylic acids were successfully converted to esters. The peaks appearing approximately at the $1635 \mathrm{~cm}^{-1}$ region of the $4 \mathrm{HCA}$, the P4HCA and the PCL-coP4HCA polymer spectra can be attributed to the doubly bonded carbon atoms $(\mathrm{C}=\mathrm{C})$ of the cinnamoyl groups that remained unchanged during the high-temperature polycondensation reaction. The peaks at $1763 \mathrm{~cm}^{-1}$ in the P4HCA and the PCL-co-P4HCA spectra were attributed to the characteristic carbonyl $(\mathrm{C}=\mathrm{O})$ stretching bands of the acetyl groups.

The chemical structures of P4HCA and PCL-co-P4HCA75 were further identified by ${ }^{1} \mathrm{H}$ NMR spectra, shown in Figure 2. The multiple peaks at $\delta_{\mathrm{a}}=7.15$ and 7.64 and at $\delta_{\mathrm{a}}=7.94$ and 6.51 p.p.m. shown in Figures $2 \mathrm{a}$ and $\mathrm{b}$ were assigned to the protons of benzene and double-carbon groups, respectively. The chemical shifts in the PCL-co-P4HCA75 spectrum $\left(\delta_{\mathrm{e}}=4.25,1.77,1.50\right.$ and 2.50 p.p.m. $)$ can be attributed to the different proton groups found in PCL. Moreover, the compositions of 4HCA in the copolymers can be calculated by comparing the peak area of the doubly bonded carbon atom groups in P4HCA (c) and the methylene groups in PCL (e). The

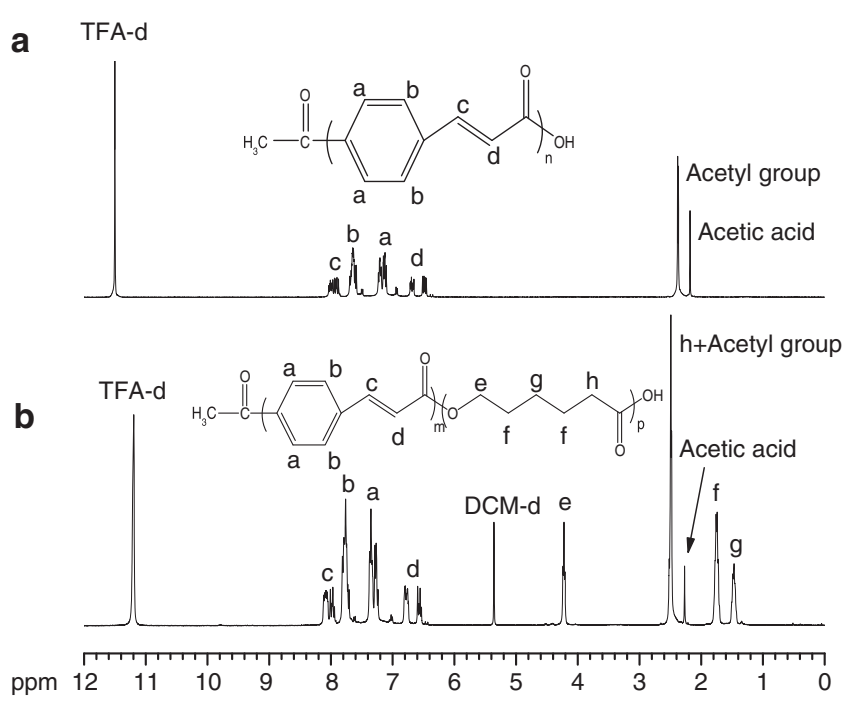

Figure $2{ }^{1} \mathrm{H} \quad$ NMR spectra of P4HCA (a) and PCL-co-P4HCA75 (b) polymers.

obtained results are shown in Table 1 and correspond well to the feed amounts. These results suggested a successful preparation of the PCL-co-P4HCA copolymers.

\section{Photoreactivity}

The cinnamoyl group is well known to undergo cis-trans isomerizations and inter- and intramolecular $[2+2]$ cycloaddition with UV irradiation (Figure 3a). ${ }^{17-20}$ The photoreactivity of the produced PCL-co-P4HCA copolymers was examined in THF solutions at room temperature by UV-visible spectrophotometery. Figure $3 \mathrm{~b}$ shows the changes in the UV absorption of the PCL-co-P4HCA12 polymer during UV irradiation at $\lambda=302 \mathrm{~nm}$ as a function of irradiation time. The absorption of the cinnamoyl group at $306 \mathrm{~nm}$ decreased rapidly within 15 min of UV irradiation, and then decreased gradually with increasing irradiation time. This phenomenon indicated that the photoreaction (crosslinking) occurred rapidly at the early stages of UV irradiation, where there was a high initial concentration of cinnamoyl groups. Other PCL-co-P4HCA copolymers with different 4HCA compositions showed the same photoreactive behavior during UV irradiation, as shown in Figure 3c. PCL-co-P4HCA copolymers with higher 4HCA compositions showed greater photoreactivity rates. The crosslinking degree of PCL-co-P4HCA copolymers was also reduced with a decrease in $4 \mathrm{HCA}$ content. 

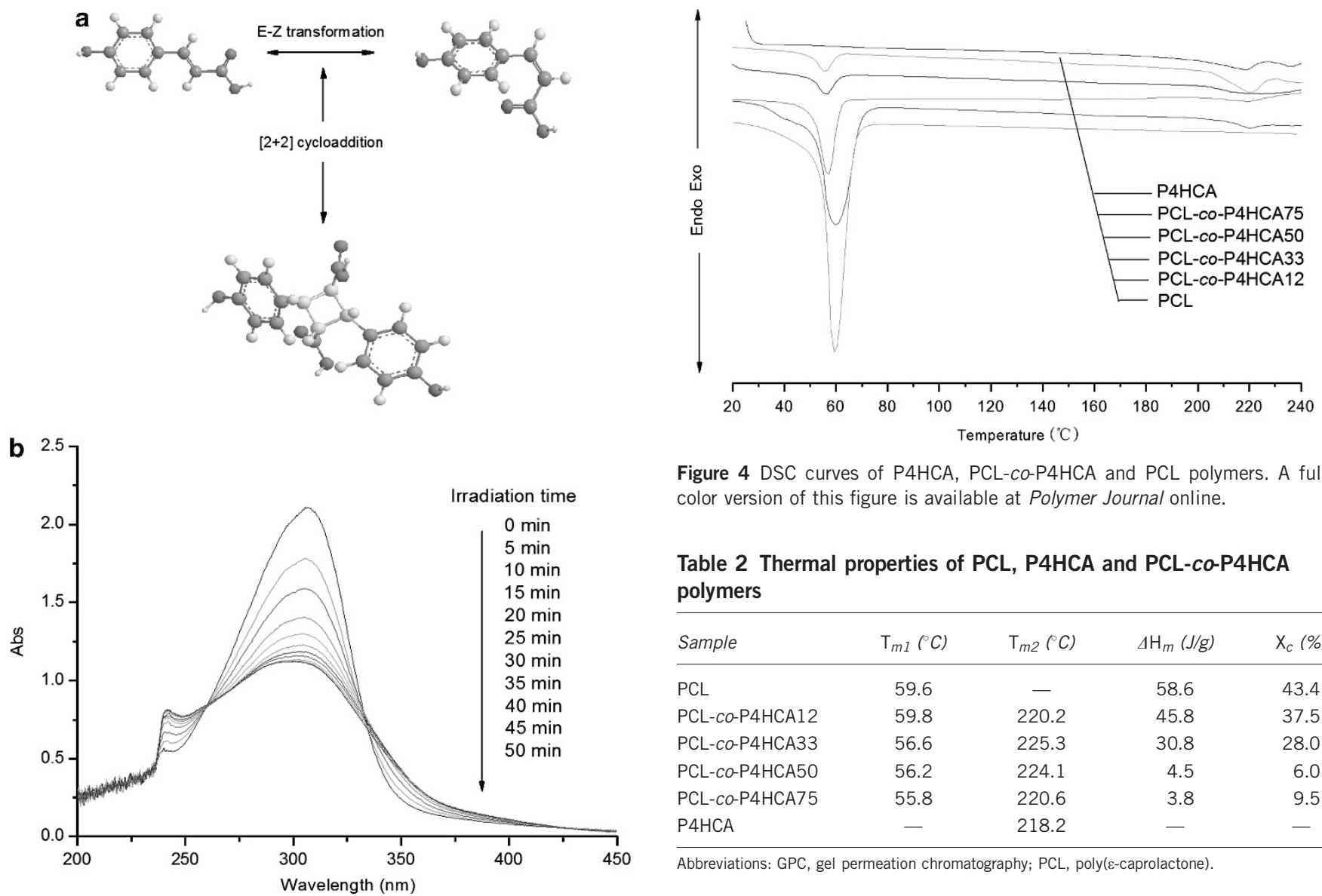

Figure 4 DSC curves of P4HCA, PCL-Co-P4HCA and PCL polymers. A full color version of this figure is available at Polymer Journal online.

Table 2 Thermal properties of PCL, P4HCA and PCL-co-P4HCA polymers

\begin{tabular}{lcccc}
\hline Sample & $\mathrm{T}_{m 1}\left({ }^{\circ} \mathrm{C}\right)$ & $\mathrm{T}_{m 2}\left({ }^{\circ} \mathrm{C}\right)$ & $\Delta \mathrm{H}_{m}(\mathrm{~J} / g)$ & $\mathrm{X}_{c}(\%)$ \\
\hline PCL & 59.6 & - & 58.6 & 43.4 \\
PCL-Co-P4HCA12 & 59.8 & 220.2 & 45.8 & 37.5 \\
PCL-co-P4HCA33 & 56.6 & 225.3 & 30.8 & 28.0 \\
PCL-Co-P4HCA50 & 56.2 & 224.1 & 4.5 & 6.0 \\
PCL-co-P4HCA75 & 55.8 & 220.6 & 3.8 & 9.5 \\
P4HCA & - & 218.2 & - & - \\
\hline
\end{tabular}

Abbreviations: GPC, gel permeation chromatography; PCL, poly( $\varepsilon$-caprolactone).

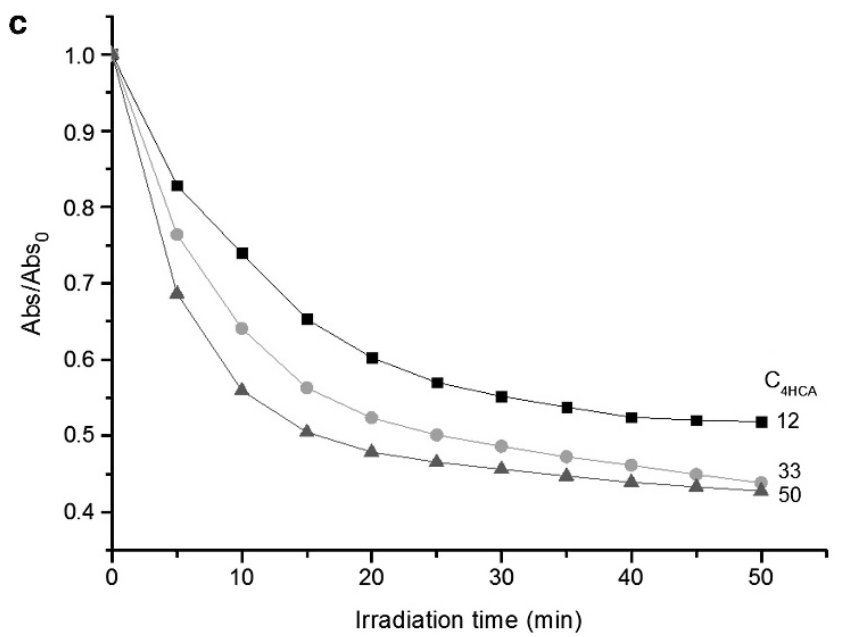

Figure 3 (a) Reaction schematic of the E-Z transformation and photodimerization of two forms of $4 \mathrm{HCA}$ into its dimer linked by cyclobutane groups. (b) UV-vis spectra of PCL-co-P4HCA12 in THF during UV irradiation at $\lambda=302 \mathrm{~nm}$. (c) Absorption $\left(\lambda_{\max }=306 \mathrm{~nm}\right)$ changes of PCL-co-P4HCA copolymers during UV irradiation at $\lambda=302 \mathrm{~nm}$. A full color version of this figure is available at Polymer Journal online.

\section{Thermal properties}

The thermal properties of PCL, P4HCA and PCL-co-P4HCA were investigated by DSC (Figure 4). DSC analyses were performed twice to remove the previous thermal history of the samples. The heating curves of P4HCA and PCL each showed a solitary melting endotherm

peak $\left(T_{\mathrm{m}}\right)$, which occurred at 218.2 and $59.5^{\circ} \mathrm{C}$, respectively. These two melting endotherm peaks appeared in the heating curves of the PCL-co-P4HCA copolymers. The phase transition temperatures and heat of fusion $\left(\Delta H_{\mathrm{m}}\right)$ of the polymer samples are summarized in Table 2. The relative crystallinity of PCL chain in the polymers (Xc) could be estimated with respect to PCL content in the polymer using the following formula:

$$
X_{\mathrm{c}}(\%)=100 \times \Delta H_{\mathrm{m}} / \omega \times \Delta H_{\mathrm{m}}^{0}
$$

where $\Delta H_{\mathrm{m}}^{0}$ is the heat of fusion of $100 \%$ crystalline of the PCL homopolymer $\left(135 \mathrm{Jg} \mathrm{g}^{-1}\right),{ }^{21} \Delta H_{\mathrm{m}}$ is the fusion heat of the sample and $\omega$ is the weight fraction of the PCL in the polymer. With the increase in PCL chains in the PCL-co-P4HCA polymers, the melting endotherm peak at $55-60{ }^{\circ} \mathrm{C}$ increased. The $T_{\mathrm{m} 1}$ and $X_{\mathrm{c}}$ of PCL-coP4HCA polymers were almost lower than the ones of pure PCL, and the results illustrated that the 4HCA chain disturbed the crystal growth of PCL compositions.

Table 3 showed the thermal stability of P4HCA, PCL and PCL-coP4HCA polymers. The half-decomposition temperatures $\left(T_{1 / 2}\right)$ of P4HCA and PCL were 575 and $406{ }^{\circ} \mathrm{C}$, respectively, and the $T_{1 / 2}$ of the PCL-co-P4HCA copolymers decreased with a decrease in the $4 \mathrm{HCA}$ content in the copolymers. The residual sample weight of these polymers at $500{ }^{\circ} \mathrm{C}\left(\omega_{500}\right)$ showed a similar trend. These results demonstrate that the P4HCA and the PCL-co-P4HCA polymers possess excellent thermal stabilities that are induced by the highly regular and abundant rigid benzene rings, as well as the conjugated structure formed by the benzene rings and the double-carbon bonds in the P4HCA chains. Moreover, the thermal stabilities of the P4HCA 
Table 3 Thermal stabilities of PCL, P4HCA and PCL-co-P4HCA polymers

\begin{tabular}{lccccc}
\hline & \multicolumn{3}{c}{ Before UV } & & \multicolumn{2}{c}{ After UV } \\
\cline { 2 - 3 } Sample & $\mathrm{T}_{1 / 2}\left({ }^{\circ} \mathrm{C}\right)$ & $\omega_{500}(\%)$ & & $\mathrm{T}_{1 / 2}\left({ }^{\circ} \mathrm{C}\right)$ & $\omega_{500}(\%)$ \\
\hline P4HCA & 575 & 56.1 & & 523 & 51.8 \\
PCL-Co-P4HCA75 & 444 & 42.5 & & 411 & 40.8 \\
PCL-Co-P4HCA50 & 417 & 35.3 & & 405 & 30.5 \\
PCL-Co-P4HCA33 & 411 & 21.2 & & 404 & 13.8 \\
PCL-Co-P4HCA12 & 406 & 13.1 & & 402 & 7.0 \\
PCL & 406 & 6.3 & & - & -
\end{tabular}

Abbreviation: $\mathrm{PCL}$, poly( $\varepsilon$-caprolactone)

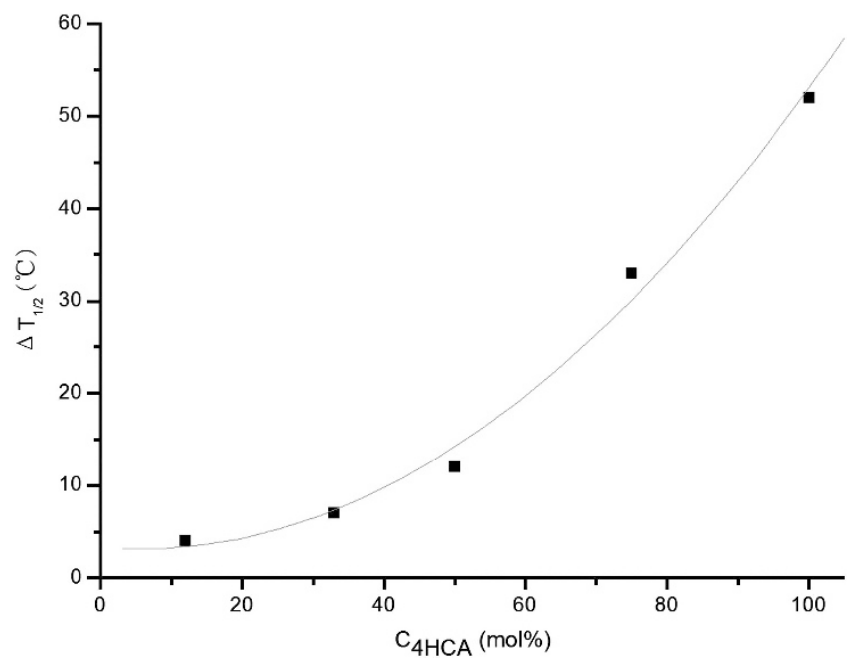

Figure 5 The effect of $4 \mathrm{HCA}$ composition on $\Delta T_{1 / 2}$ of $\mathrm{PCL}-\mathrm{Co}-\mathrm{P} 4 \mathrm{HCA}$ copolymers before and after UV irradiation. A full color version of this figure is available at Polymer Journal online.

and PCL-co-P4HCA polymers decrease when irradiated with UV rays, which may be possibly due to the change of the copolymer structure. Isomerization and [2+2] cycloaddition of the cinnamoyl groups occurred under UV irradiation, which destroyed the regularity and the conjugated structure of the copolymers. In addition, the bond energy of the carbon-carbon double bond was much higher than the bond energy of the carbon-carbon single bond in the four-ring structure, which may also lead to a decrease in the thermal stabilities of the copolymers. A comparison of the $\mathrm{T}_{1 / 2}$ between the PCL-coP4HCA copolymers before and after UV irradiation allowed for the obtaining of $\Delta T_{1 / 2}$, which is shown in Figure 5 as being dependent on $4 \mathrm{HCA}$ content in the copolymer. The PCL-co-P4HCA copolymers with a higher $4 \mathrm{HCA}$ content showed larger $\Delta T_{1 / 2}$ values due to the higher degree of photoreactivity. These results indicated that the photoreaction would significantly affect the thermal stabilities of the copolymers.

\section{Fluorescence properties}

The polymers based on cinnamic acid derivatives have fluorescence properties. $^{22,23}$ The fluorescence emission spectra were obtained at an excitation wavelength of $375 \mathrm{~nm}$. The characteristic fluorescence emission peaks of P4HCA and PCL-co-P4HCA polymers are shown in Figure 6, which exhibit broad and strong emission peaks at the

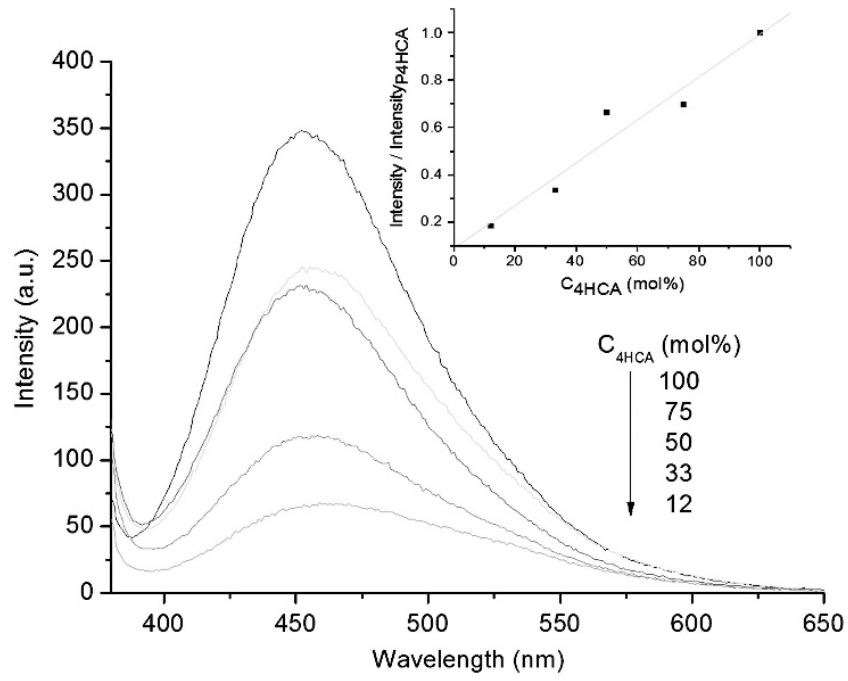

Figure 6 Fluorescence emission spectra of P4HCA and PCL-co-P4HCA polymers. The inset shows how the fluorescent intensity changes with $4 \mathrm{HCA}$ composition. A full color version of this figure is available at Polymer Journal online.

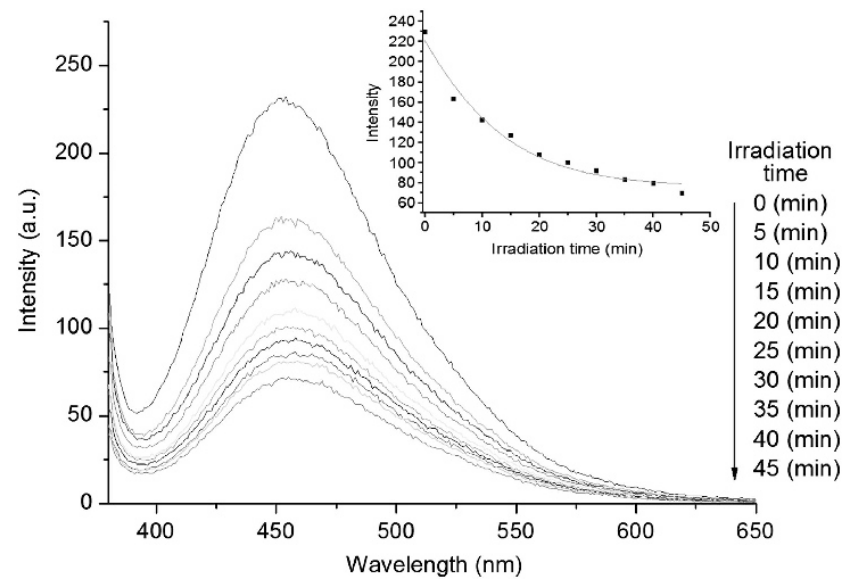

Figure 7 Fluorescence emission spectra of PCL-Co-P4HCA50 with UV irradiation at $\lambda=302 \mathrm{~nm}$ for various times, and the inset shows how the fluorescence intensity changes with UV irradiation time. A full color version of this figure is available at Polymer Journal online.

$400-650 \mathrm{~nm}$ region corresponding to the benzene rings and the conjugated system of $\mathrm{C}=\mathrm{C}$ double bonds within the polymers. The maximum emission peak intensity at $450 \mathrm{~nm}$ of these polymers decreased with a decrease in 4HCA content, and a linear relationship was found between the peak intensity and the composition of 4HCA in the polymers (inset in Figure 6). It was also confirmed that the reaction between 4HCA and PCL was successful. Figure 8 shows the fluorescence emission spectra of PCL-co-P4HCA50 after UV irradiation at $\lambda=302 \mathrm{~nm}$ over a given period of time. The fluorescent intensity decreased with an increasing irradiation time (inset in Figure 7), which was similar to the previous results obtained from UV absorption tests. This phenomenon indicated that the cinnamoyl group could undergo photoreaction in the absence of a photo initiator, and the degree of conjugation within the polymers was reduced.

\section{Crystallinity}

The crystallizations of P4HCA and PCL-co-P4HCA polymers were characterized by WXRD measurements, as shown in Figure 8. P4HCA 


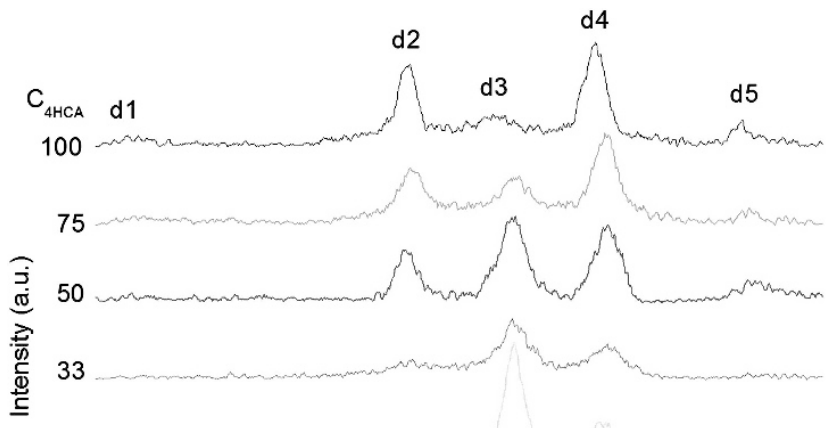

12

0

10

20

$2 \theta$ (deg)

Figure 8 WXRD diagrams of P4HCA, PCL-Co-P4HCA and PCL polymers. A full color version of this figure is available at Polymer Journal online.

showed five diffraction peaks at $2 \theta=11.0^{\circ}(\mathrm{d} 1), 18.6^{\circ}(\mathrm{d} 2), 21.0^{\circ}$ $(\mathrm{d} 3), 23.8^{\circ}(\mathrm{d} 4)$ and $27.8^{\circ}(\mathrm{d} 5)(\theta=$ diffraction angel) consistent with the results presented by Kaneko et al., ${ }^{24}$ and PCL showed two sharp diffraction peaks at $2 \theta=21.4^{\circ}(\mathrm{d} 3)$ and $23.7^{\circ}(\mathrm{d} 4) .{ }^{25}$ The WXRD pattern indicates that the P4HCA and PCL homopolymers are all crystalline. The copolymerization of $4 \mathrm{HCA}$ and PCL produced a crystalline PCL-co-P4HCA copolymer. When the composition of $4 \mathrm{HCA}$ in the PCL-co-P4HCA copolymers decreased, it was observed that the intensity of crystal diffraction peaks derived from the P4HCA phase became weaker, while the peaks derived from the PCL phase became stronger.

\section{Wettability of the PCL-co-P4HCA copolymers}

Investigating the interaction between water and any materials used as biological implants is very important, which requires an evaluation of the contact angles and the water absorption capacities of the PCL-coP4HCA sheets. The contact angles of the copolymer sheet surfaces with different 4HCA compositions were compared to evaluate the surface wettability (Figure 9). The water contact angles of the PCL-coP4HCA12, PCL-co-P4HCA33 and PCL-co-P4HCA50 sheets were found to be $74 \pm 2^{\circ}, 70 \pm 3^{\circ}$ and $68 \pm 2^{\circ}$, respectively, which decreased with an increase in 4HCA content, possibly due to the high hydrophobicity of the P4HCA chains. Moreover, the contact angles of PCL-co-P4HCA sheets increased to $81 \pm 5^{\circ}, 80 \pm 2^{\circ}$ and $78 \pm 2^{\circ}$, respectively, after UV irradiation. These results suggest that crosslinking caused by UV irradiation increased the surface hydrophobicity. Therefore, it can be said that the surface wettability of the PCLco-P4HCA sheets are controlled by adjusting the chemical compositions and the conditions of UV irradiation. The water absorption of the PCL-co-P4HCA sheets in deionized water was performed for $24 \mathrm{~h}$, and the results are shown in Table 4 . The water absorption was only $0.54 \%$ of because of the hydrophobic property of the copolymer, and decreased to $0.32 \%$ after the UV irradiation. PCL-co-P4HCA12 and PCL-co-P4HCA33 sheets showed zero water absorption when put into contact with deionized water for $24 \mathrm{~h}$.

\section{Degradation behavior}

The hydrolytic degradation behaviors of the PCL-co-P4HCA sheet with and without UV irradiation were investigated using buffer solutions of $\mathrm{pH}=7.4$ and 13.3 at $37^{\circ} \mathrm{C}$. Figure 10 showed the weight

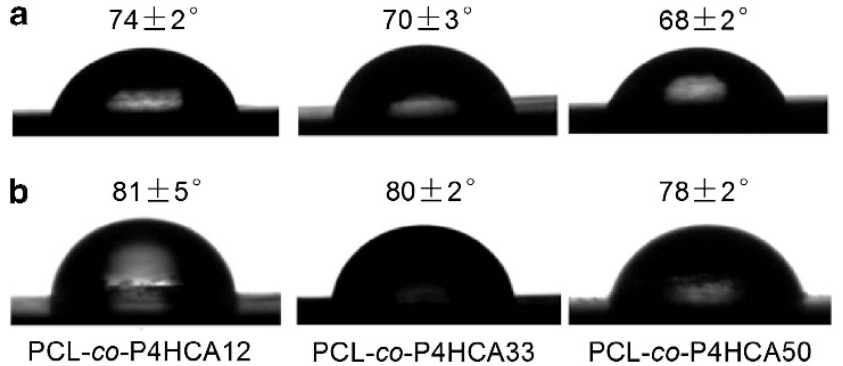

Figure 9 Contact angle images of PCL-Co-P4HCA sheets without (a) and with (b) UV irradiation for $30 \mathrm{~min}$.

Table 4 Water absorptions of the PCL-co-P4HCA sheets with and without UV irradiation

\begin{tabular}{lcc} 
Sample & Without UV & With UV irradiation for $30 \mathrm{~min}$ \\
\hline PCL-co-P4HCA12 & - & - \\
PCL-co-P4HCA33 & - & - \\
PCL-co-P4HCA50 & $0.54 \%$ & $0.32 \%$ \\
\hline
\end{tabular}

Abbreviation: UV, ultraviolet.

loss of the PCL-co-P4HCA sheets as a function of degradation time. A rapid weight loss was observed at the initial period of immersion into both solutions, but the hydrolysis rate became slower and stabilized during the latter period of degradation. The initial weight loss might be caused mainly by the degradation of the low molecular weight polymer chains. The hydrolysis rate of the PCL-co-P4HCA sheets increased with an increase in 4HCA content, and the rates of hydrolysis were significantly higher under alkaline conditions than under neutral conditions. The degradation rates of the copolymers after UV irradiation were slightly slower than the copolymers that did not undergo UV irradiation (Figure 10c and d), which was different from the results reported by Thi et al. ${ }^{20,26}$ This phenomenon might be caused by a fixed structure induced from crosslinking and the higher surface hydrophobicity of the PCL-co-P4HCA sheets.

\section{CONCLUSIONS}

PCL-co-P4HCA copolymers were synthesized successfully by the thermal polycondensation of 4HCA with PCL. The copolymers showed two melting endotherm peaks belonging to PCL and P4HCA chains, and the WXRD patterns also indicated that the copolymers were crystalline. The introduction of rigid, conjugated 4HCA polymer segments could increase the thermal stability of the copolymers, which was observed to increase with an increase in 4HCA content. The thermal stability of PCL-co-P4HCA declined upon irradiation with UV rays, and the thermal stability declined with an increase in 4HCA content. PCL-co-P4HCA copolymers showed fluorescence and photoreactive properties, and the strongest fluorescence emission peak decreased with a decrease in 4HCA content. The results of accelerated degradation tests showed that the contact angles of PCL-co-P4HCA sheets decreased and degradation rate increased with an increase in 4HCA composition within the copolymers. After UV irradiation, the contact angle increased and the degradation rate decreased. Therefore, the degradation of the copolymers could be controlled by changing the copolymer components and by introducing UV irradiation. The copolymers would have good compatibilities 

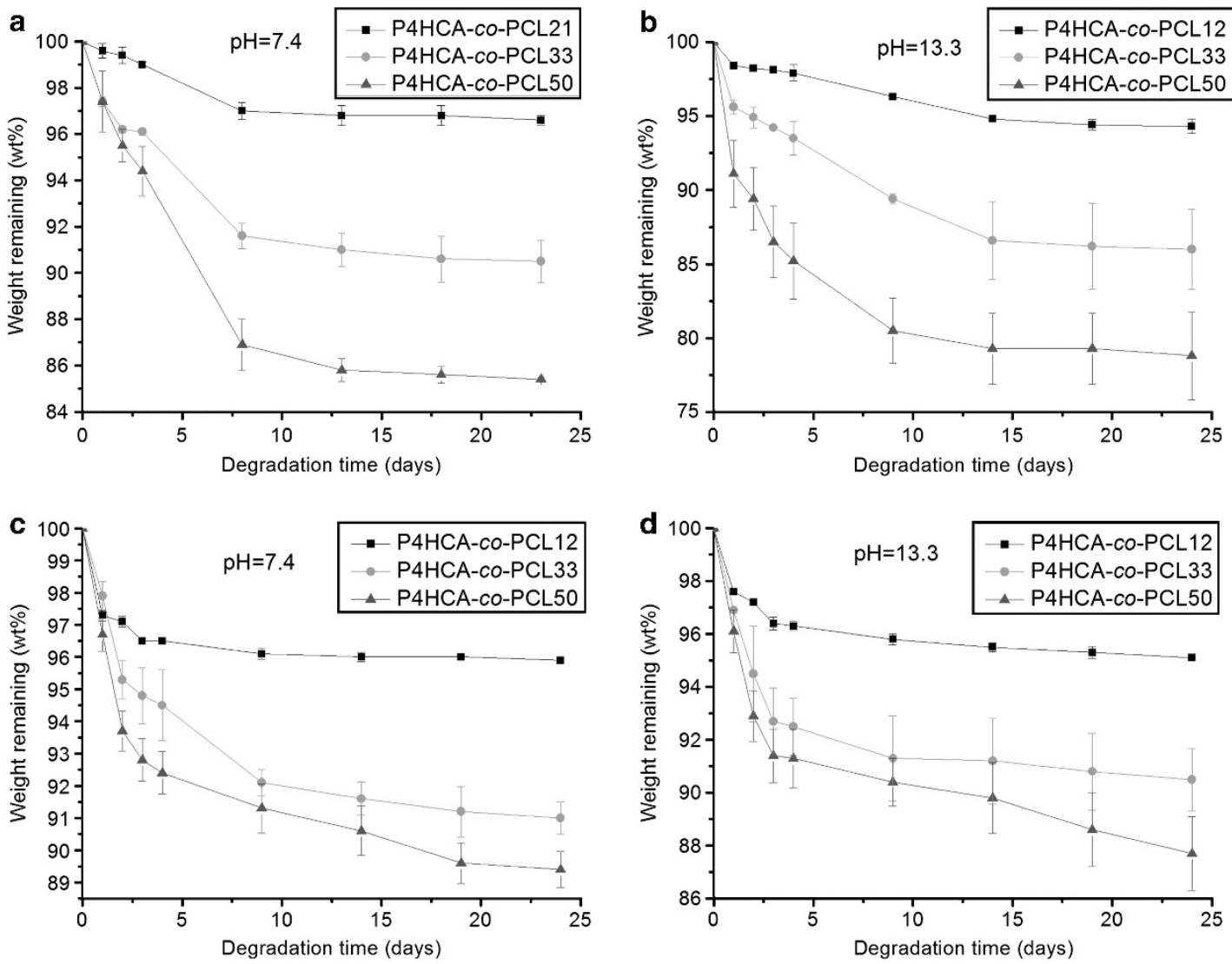

Figure 10 Degradation behaviors of PCL-Co-P4HCA polymer sheets as a function of hydrolysis time. The weight remaining percentage of PCL-Co-P4HCA polymer sheets in buffer solutions at $37^{\circ} \mathrm{C}(\mathrm{pH}=7.4$ and 13.3) (a and $\mathbf{b})$ without or (c and $\mathbf{d}$ ) with UV irradiation for 30 min. A full color version of this figure is available at Polymer Journal online.

and useful applications as novel environmental and biomedical materials.

\section{ACKNOWLEDGEMENTS}

We would like to thank the National Natural Science Foundation of China (No. 21004029, 51173072), the Research and Innovation Program for Graduate Colleges and Universities (CX10B_225Z) and the Fundamental Research Funds for the Central Universities (JUSRP11109) for financially supporting this research.

$1 \mathrm{Ma}$, X. F., Yu, J. G. \& Wang, N. Compatibility characterization of poly(lactic acid)/poly(propylene carbonate) blends. J. Polym. Sci. B: Polym. Phys. 44, 94-101 (2006).

2 Hofmann, D., Castaňo, M. E., Kratz, K. \& Lendlein, A. Knowledge-based approach towards hydrolytic degradation of polymer-based biomaterials. Adv. Mater. 21, $3237-$ 3245 (2009).

3 Chang, K. Y. \& Lee, Y. D. Ring-opening polymerization of $\varepsilon$-caprolactone initiated by the antitumor agent doxifluridine. Acta Biomater. 5, 1075-1081 (2009).

4 Yan, J. L., Ye, Z. Y, Luo, H. Y., Chen, M., Zhou, Y., Tan, W. S., Xiao, Y., Zhang, Y. \& Lang, M. D. Synthesis, characterization, fluorescence labeling and cellular internalization of novel amine-functionalized poly(ethylene glycol)-block-poly(e-caprolactone) amphiphilic block copolymers. Polym. Chem. 2, 1331-1340 (2011).

5 Kang, J. \& Beers, K. J. Macromolecular transport through nanostructured and porous hydrogels synthesized using the amphiphilic copolymer, PCL-b-PEO-b-PCL. Eur. Polym. J. 45, 3004-3009 (2009).

6 Katsumata, K., Saito, T., Yu, F., Nakamura, N. \& Inoue, Y. The toughening effect of a small amount of poly( $\varepsilon$-caprolactone) on the mechanical properties of the poly(3-hydroxybutyrate-co-3-hydroxyhexanoate)/PCL blend. Polym. J. 43, 484-492 (2011).

7 Calandrelli, L., Calarco, A., Laurienzo, P., Malinconico, M., Petillo, O. \& Peluso, G. Compatibilized polymer blends based on PDLLA and PCL for application in bioartificial liver. Biomacromolecules 9, 1527-1534 (2008).
8 Mikhail, A. S. \& Allen, C. Poly(ethylene glycol)-b-poly(e-caprolactone) micelles containing chemically conjugated and physically entrapped docetaxel: synthesis, characterization, and the influence of the drug on micelle morphology. Biomacromolecules 11, 1273-1280 (2010)

9 Xu, F. J., Zheng, Y. Q., Zhen, W. J. \& Yang, W. T. Thermoresponsive poly(N-isopropyl acrylamide)-grafted polycaprolactone films with surface immobilization of collagen. Colloid Surface B. 85, 40-47 (2011).

10 Li, C. Z., Gu, C. H., Zhang, Y. \& Lang, M. D. Synthesis and self-assembly of $\mathrm{pH}$-responsive amphiphilic poly( $\varepsilon$-caprolactone)-block-poly(acrylic acid) copolymer. Polym. Bull 68, 69-83 (2012).

11 Basavaraja, C., Kim, D. G., Kim, W. J., Kim, J. H. \& Huh, D. S. Morphology and charge transport properties of chemically synthesized polyaniline-poly( $\varepsilon$-caprolactone) polymer films. Bull. Korean Chem. Soc. 32, 927-933 (2011).

12 Kaneko, T. High-performance functional ecopolymers based on flora and fauna. Chem. Rec. 7, 210-219 (2007)

13 Kaneko, T., Thi, T. H., Shi, D. J. \& Akashi, M. Environmentally degradable, highperformance thermoplastics from phenolic phytomonomers. Nat. Mater. 5, 966-970 (2006).

14 Matsusaki, M., Kishida, A., Stainton, N., Ansell, C. W. G. \& Akashi, M. Synthesis and characterization of novel biodegradable polymers composed of hydroxycinnamic acid and D,L-Lactic acid. J. Appl. Polym. Sci. 82, 2357-2364 (2001).

15 Kaneko, T., Thi, T. H., Matsusaki, M. \& Akashi, M. Biodegradable LC oligomers with cranked branching points form highly oriented fibrous scaffold for cytoskeletal orientation. Chem. Mater. 18, 6220-6226 (2006).

16 Matsusaki, M., Thi, T. H., Kaneko, T. \& Akashi, M. Enhanced effects of lithocholic acid incorporation into liquid-crystalline biopolymer poly(coumaric acid) on structural ordering and cell adhesion. Biomaterials 26, 6263-6270 (2005).

17 Nagata, M. \& Inaki, K. Biodegradable and photocurable multiblock copolymers with shape-memory properties from poly(e-caprolactone) diol, poly(ethylene glycol), and 5-cinnamoyloxyisophthalic acid. J. Appl. Polym. Sci. 120, 3556-3564 (2011).

18 Nagata, M. \& Hizakae, S. Synthesis and characterization of photocrosslinkable biodegradable polymers derived from 4-hydroxycinnamic acid. Macromol. Biosci. 3, 412-419 (2003).

19 Yasaki, K., Suzuki, T., Yazawa, K., Kaneko, D. \& Kaneko, T. Effects of double photoreactions on polycoumarate photomechanics. J. Polym. Sci. A: Polym. Chem. 49, 1112-1118 (2011).

20 Thi, T. H., Matsusaki, M. \& Akashi, M. Photoreactive polylactide nanoparticles by the terminal conjugation of biobased caffeic acid. Langmuir. 25, 10567-10574 (2009). 
21 Hoff, W. D., Dux, P., Hard, K., Devreese, B., Nugteren Roodzant, I. M., Crielaard, W. Boelens, R., Kaptein, R., Beeumen, J. V. \& Hellingwer, K. J. Thiol ester-linked p-coumaric acid as a new photoactive prosthetic group in a protein with rhodopsin-like photochemistry. Biochemistry 33, 13959-13962 (1994).

22 Kan, K., Kaneko, D. \& Kaneko, T. Polarized emission of wholly aromatic bio-based copolyesters of a liquid crystalline nature. Polymers 3, 861-874 (2011).

23 Dong, W. F., Li, H. L., Chen, M. Q., Ni, Z. B., Zhao, J. S., Yang, H. P. \& Gijsman, P. Biodegradable bio-based polyesters with controllable photo-crosslinkability, thermal and hydrolytic stability. J. Polym. Res. 18, 1239-1247 (2011).
24 Kaneko, T., Matsusaki, M., Thi, T. H. \& Akashi, M. Thermotropic liquid-crystalline polymer derived from natural cinnamoyl biomonomers. Macromol. Rapid. Commun. 25, 673-677 (2004).

25 Allo, B. A., Rizkalla, A. S. \& Mequanint, K. Synthesis and electrospinning of $\varepsilon$-polycaprolactone-bioactive glass hybrid biomaterials via a sol-gel process. Langmuir. 26, 18340-18348 (2010).

26 Thi, T. H., Matsusaki, M. \& Akashi, M. Develop of photoreactive degradable branched polyesters with high thermal and mechanical properties. Biomacromolecules 10 , 766-772 (2009) 